\title{
Study on the method of intermittent chaos to detect the weak signal
}

\author{
Song Xu-dong ${ }^{1, a}$, Chen $x^{2}{ }^{2, b}$ \\ ${ }^{1}$ 91338th, The People's Liberation Army of China, Zhanjiang 524022, China \\ ${ }^{2}$ Northwestern Ploytechnical University, Xi'an 710072, China

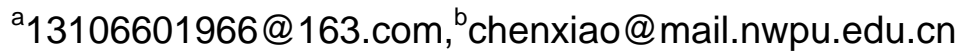

Keywords: Duffing oscillator; weak signal detection; intermittent chaos; chaotic oscillator array.

\begin{abstract}
In this paper, we use the largest Lyapunov exponent proved that Duffing oscillators system have the immune to arbitrary distribution with zero-mean noise. For the unknown signal, we can use intermittent chaos to realize signal detection. Through experiment simulation, we use the least number of chaotic oscillator array to realize the signal frequency detection. Simulation experiments show that this method is simple, effective, high detection precision.
\end{abstract}

\section{Introduction}

Weak signal detection technology is an emerging technology and an important part of modern information theory which extracts the useful weak signal from the strong noise based on the theory of modern electronics and signal processing methods. Here, the 'weak signal' generally refers to a signal that has a low SNR. Typically, when the SNR is lower than -10dB, using conventional methods would be difficult to extract the characteristic of the useful signal from the background noise ${ }^{[1]}$.In addition, when the background noise present to be non-stationary and no-Gaussian ,the method like matched filtering, coherent accumulation ,the optimal detection can't do well at signal detection $^{[2-4]}$.

Donald L.Birx ${ }^{[5]}$ had the first attempt to detect weak signal by using chaotic oscillators in 1992.The basic principle of chaotic oscillator detection of weak signal is the properties which are sensitive to certain signals and immune to noise at the same time ${ }^{[6]}$. Ideally, using Duffing chaotic oscillator to detect sinusoidal signal under the background of white noise, the detection signal to noise ratio up to $-60 \mathrm{~dB}^{[7]}$, for a square wave signal, in any colored noise detection signal to noise ratio up to - $48 \mathrm{~dB}{ }^{[8]}$, For the detection of weak signals in strong background noise, Wang Yongsheng ${ }^{[9]}$ proposed through the selection of input noise and simulation step size to improve the detection performance. In practical applications, for unknown frequency weak signal detection how to get the frequency of signal is the key parameter, now using chaotic oscillator array to detection signal is an effective method ${ }^{[10]}$.

In this paper, based on the analysis of the theory of using Duffing oscillator detect signal . we use the largest Lyapunov exponent proved that Duffing oscillators system have the immune to arbitrary distribution with zero-mean noise . For unknown frequency signal, we use the least number of chaotic oscillator array to realize the signal frequency detection. Simulation experiments show that this method is simple, effective, high detection precision. In section II,the theory of using Duffing oscillator detect signal is presented ,and also proved that Duffing oscillators system have the immune to arbitrary distribution with zero-mean noise. In section III, we discuss the intermittent chaos theory. In section IV,we analysis the method of using chaotic oscillator array to realize the signal frequency detection, at the same time ,simulation were performed under low SNR. Conclusion will be give in section $\mathrm{V}$.

\section{Fundamental principles for using Duffing oscillator in signal frequency detection}

\section{Duffing chaotic oscillator}

We consider a nonlinear elastic system with symmetry elastic potential energy, its potential function 


$$
\mathrm{U}(\mathrm{x})=-\mathrm{x}^{2} / 2+\mathrm{x}^{4} / 4
$$

It has two minima $(x= \pm 1)$, a maximum point $(x=0)$, so it can form a bi-stable potential well, as shown in Fig.1,it can be understood as: a track of potential field, the particle move between the left and right in potential well, its trajectory is $\mathrm{x}(\mathrm{t})$, per unit mass of the particles by the external field force $F(t)$, the damping force $k \dot{x}$, potential field force on the particle $d U(x) / d x$, its motion equation can be express as

$$
\ddot{\mathrm{x}}(\mathrm{t})+\mathrm{k} \dot{\mathrm{x}}(\mathrm{t})+\mathrm{dU}(\mathrm{x}) / \mathrm{dx}=\mathrm{F}(\mathrm{t})
$$

When the external force field $\mathrm{F}(\mathrm{t})$ is periodic driving force with angular frequency $\omega$, the amplitude $\gamma$,equation (2) becomes

$$
\ddot{x}(\mathrm{t})+\mathrm{kx}(\mathrm{t})-\mathrm{x}(\mathrm{t})+\mathrm{x}^{3}(\mathrm{t})=\gamma \cos \omega \mathrm{t}
$$

The equation (3) Called homes Duffing equation ${ }^{[11]}$. where $k$ is the damping ratio, $-x(t)+x^{3}(t)$ is a non-linear restoring force. Because of the nonlinear terms, Duffing equation (3) has the characteristics of nonlinear dynamics ${ }^{[12]}$, is one of the common model to research the chaotic motion. In the case of a fixed $\mathrm{k}$, with the increasing of $\gamma$ the system experience the homoclinic orbit, cycle bifurcation ,chaotic trajectories, critical periods, large scale periodic state .as Fig.2(a) show the critical state of chaos, Fig.2 (b)show Large scale cycle state.

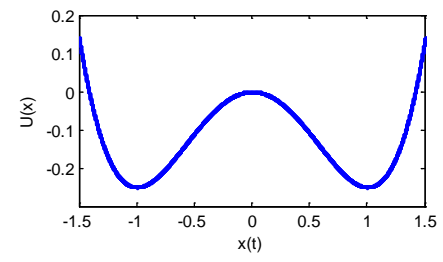

Fig.1 potential function of bistable system

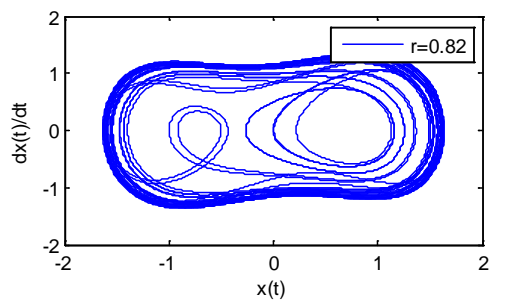

(a) $\mathrm{k}=0.5$ Critical state of chaos

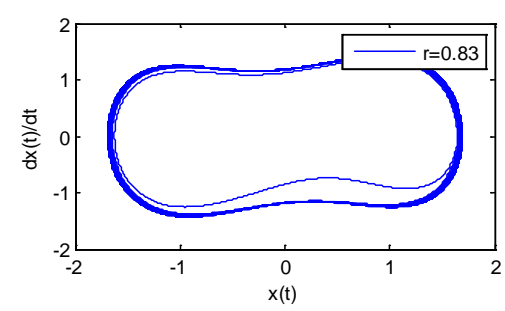

(b) $\mathrm{k}=0.5$ Large scale cycle state

Fig .2 The phase trajectory of Duffing system

For the purpose of detect weak signals with different frequency. we do the frequency transformation .Defining $\mathrm{t}=\omega \tau$, we obtain

And add the input signal S(t), we obtain

$$
\frac{1}{\omega^{2}} \ddot{x}(\omega \tau)+\frac{k}{\omega} \dot{x}(\omega \tau)-x(\omega \tau)+x^{3}(\omega \tau)=\gamma \cos \omega \tau
$$

$$
\left\{\begin{array}{l}
\dot{\mathrm{x}}=\omega \mathrm{y} \\
\dot{\mathrm{y}}=\omega\left(-\mathrm{k} \cdot \mathrm{y}+\mathrm{x}-\mathrm{x}^{3}+\gamma \cos \omega \mathrm{t}+\mathrm{S}(\mathrm{t})\right)
\end{array}\right.
$$

Where $S(t)=s(t)+n(t), s(t)=A \cos ((\omega+\Delta \omega) t+\varphi), n(t) \sim N\left(0, \delta^{2}\right)$

\section{The immunity of noise to Duffing chaotic oscillator}

Form[1],we know that in the statistical sense, any zero-mean noise does not affect the system of the original trajectory, just make the system trajectory becomes rough and fluctuations in the vicinity of the ideal trajectory, and the result also applies to any distribution.

Then we calculate the largest Lyapunov exponent when adding different intensity noise.where the Duffing oscillator parameters $\mathrm{k}=0.5, \omega=1 \mathrm{rad} / \mathrm{s}$. At the critical state of chaos ,we add different noise.(a) white noise: $\mathrm{n}(\mathrm{t}) \sim \mathrm{N}\left(0, \delta^{2}\right)$;(b) Gaussian color noise: using gaussian white noise through a fourth-order bandpass filter to produce Gaussian color noise. the center frequency of the filter is $350 \mathrm{~Hz}$, the bandwidth of $100 \mathrm{~Hz}$ (presumably through a Gaussian filter color noise cn (t), added to the system of Gaussian colored noise is $\mathrm{Cn}(\mathrm{t})=\mathrm{m} * \mathrm{cn}(\mathrm{t}))$. From the above theoretical analysis of knowledge: the case of (b) and (c) does not affect the system. For different situations, 
the largest Lyapunov exponent coefficient output Duffing time series shown in Table 1.

Table 1 largest lyapunov exponent of different noise

\begin{tabular}{|l|l|l|l|l|}
\hline Input signal & \multicolumn{2}{|l|}{$\mathrm{n}(\mathrm{t})$} & \multicolumn{2}{l|}{$\mathrm{Cn}(\mathrm{t})$} \\
\hline parameters & $\delta^{2}=0.2$ & $\delta^{2}=9$ & $\mathrm{~m}=1$ & $\mathrm{~m}=10$ \\
\hline Largest lyapunov exponent & 0.2148 & 0.2148 & 0.1799 & 0.2826 \\
\hline
\end{tabular}

\section{Intermittent chaos}

Chaos system which constituted by Duffing oscillator is extremely sensitive to initial conditions. As was introduced in Section 2, intermittent chaos will take place if the frequency difference is small enough. Now, let us analyze this phenomenon in detail. As we can see the total periodic force of the Duffing oscillator is

$$
\begin{aligned}
\Upsilon(\mathrm{t}) & =\gamma_{\mathrm{c}} \cos (\omega \mathrm{t})+A \cos ((\omega+\Delta \omega) \mathrm{t}+\varphi) \\
& =\gamma_{\mathrm{c}} \cos (\omega \mathrm{t})+A \cos (\omega \mathrm{t}) \cos (\Delta \omega \mathrm{t}+\varphi)-\mathrm{A} \sin (\omega \mathrm{t}) \sin (\Delta \omega \mathrm{t}+\varphi) \\
& =\left(\gamma_{\mathrm{c}}+A \cos (\Delta \omega \mathrm{t}+\varphi)\right) \cos (\omega \mathrm{t})-\mathrm{A} \sin (\omega \mathrm{t}) \sin (\Delta \omega \mathrm{t}+\varphi) \\
& =\gamma(\mathrm{t}) \cos (\omega \mathrm{t}+\theta(\mathrm{t}))
\end{aligned}
$$

where $\gamma(\mathrm{t})=\sqrt{\gamma_{\mathrm{c}}{ }^{2}+2 \gamma_{\mathrm{c}} \cos (\Delta \omega \mathrm{t}+\varphi)+\mathrm{A}^{2}}, \quad \theta(\mathrm{t})=\operatorname{arctg} \frac{\mathrm{A} \sin (\Delta \omega \mathrm{t}+\varphi)}{\gamma_{\mathrm{c}}+\mathrm{A} \cos (\Delta \omega \mathrm{t}+\varphi)}, \quad \gamma_{\mathrm{c}}$ is threshold condition when the chaotic system is in a critical state. In general, $A \ll \gamma_{c}$. Thus, $\theta(t)$ is very small and its impact on system dynamica should be ignored.

From the above calculation, we can draw the following conclusions.

(1) If $\Delta \omega=0, \varphi$ plays a decisive role in the motion of the system. When $\pi-\arccos \left(\mathrm{A} / 2 \gamma_{\mathrm{c}}\right) \leq \varphi \leq$ $\pi+\arccos \left(A / 2 \gamma_{c}\right)$, we can get $\Upsilon(t) \leq \gamma_{c}$, the system still remains in chaotic motion. Only if $\varphi$ is not in this regime can the transition take place.

(2) If $\Delta \omega \neq 0, \Upsilon(\mathrm{t})$ will be periodically less than or more than the critical value $\gamma_{\mathrm{c}}$, and the cycle time $\mathrm{T}=2 \pi / \Delta \omega$, the system changes to periodic state. This is why the intermittent chaotic phenomenon takes place, and we can know the signal frequency exactly by the measurement of T. Furthermore, by determining when the transition took place, some information about the primary phase of the external signal can be known.

(3) If $\Delta \omega$ is large, making the power of the system is changing rapidly, so that the system could not respond timely. In order to make phase change of system occurred, the driving force must be applied enough. When $\gamma(t)>\gamma_{c}$, the driving force would decay very quickly, so that periodic motion could not be sustained. While, if $\gamma(t)<\gamma_{c}$, the driving force will increase quickly, and then chaotic motion cannot be last, thus an irregular intermittent chaotic would generate.

\section{Using An array of chaotic oscillator to detect the unknown frequency signal}

\section{The array of chaotic oscillator}

For the detection of the signal, the frequency of which is unknown, an array of uncoupled Duffing oscillators is used. The weak signal will act on every oscillator respectively. On the basis of the frequency transformation approach, the array can cover the whole frequency domain. If the external signal is fed into the array, the array can search and classify in the frequency domain, the signal is finally extracted from the noise, and the frequency is determined.

The oscillators' frequencies are restricted within a range from 1 to 10 , which we denote as $\Omega^{\mathrm{T}}=\left[\omega_{1}, \omega_{2}, \omega_{3}, \ldots \omega_{\mathrm{k}}\right]$.we can make it an equal-ratio array,

The array is made up of these 79 oscillators.

$$
\omega 1=1, \omega 2=1.03, \cdots \omega_{\mathrm{k}}=1.03 \omega_{\mathrm{k}-1}
$$

If the signal frequency is between 1-10,it is certain that regular intermittent chaos will take place at only to adjacent oscillators, such as oscillator $\mathrm{k}$ and $\mathrm{k}+1$.others are still in complete chaotic motion. Therefore, $\omega$ must between $\omega_{\mathrm{k}}$ and $\omega_{\mathrm{k}+1}$. Furthernore, we can measure the cycle time of the intermittent chaos and determine $\omega$ accurately.

$$
\omega=\frac{\left(\omega_{\mathrm{k}}+\Delta \omega_{\mathrm{k}}\right)+\left(\omega_{\mathrm{k}+1}-\Delta \omega_{\mathrm{k}+1}\right)}{2}
$$


where $\Delta \omega_{\mathrm{k}}=2 \pi / \mathrm{T}_{\mathrm{k}}$. As for period signals, if the signal frequency beyond 1-10, we can use a adjustment factor to the frequency of the driving force, So as to satisfy any frequency signal detection.

\section{Theory simulation}

Assuming that the input signal to be measured under the strong noise background of the system for which $s(t)=A \cos (\omega t), n(t) \sim N\left(0, \delta^{2}\right)$. As show in Fig 3, When $\omega=3 \mathrm{rad} / \mathrm{s}, A=0.07, \delta=0.3$, $\mathrm{SNR}=-15.6 \mathrm{~dB}$. The input signal-to-noise ratio is given by $\mathrm{SNR}=10 \log \left(\mathrm{A}^{2} /\left(2 \delta^{2}\right)\right)$. Use the adjacent chaotic oscillator detection test signal frequency, then the 37 th and 38th into a stable oscillator intermittent chaotic state, then we can estimate the signal frequency.
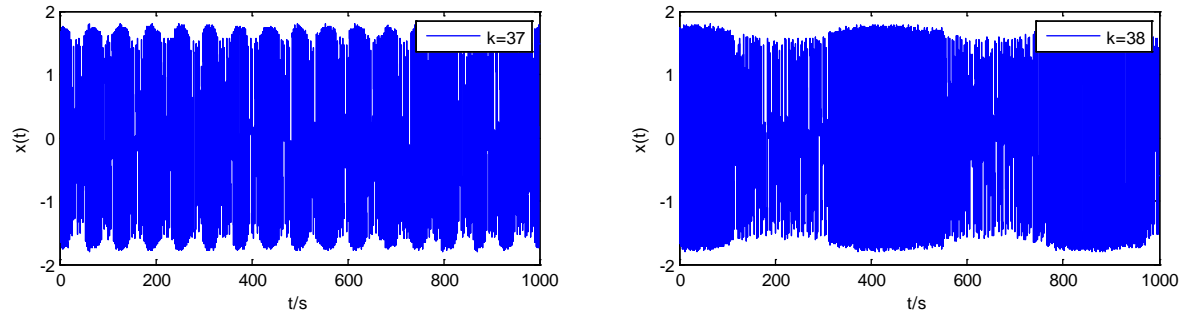

Fig.3 the intermittent chaos when using chaotic oscillator

\section{Conclusion}

In this paper, we use the largest Lyapunov exponent to confirm the Duffing chaotic system has immunity to zero-mean random noise. and also use the least number of chaotic oscillator array to realize the signal frequency detection. Simulation results show that: the method is simple and easy to implement, and its high accuracy.

\section{References}

[1] Li Yue, Yang Baojun: Chaotic Oscillator Detection Introduction, edtied by Publishing House of Electronics Industry, Beijing(2004).

[2] Gao Jinzhan: Weak Signal Detection, edtied by Tsinghua University Press, Beijing(2005).

[3] Zhou Kaibo, Dou Chengquan, Chen Tao. Journal of WUT. Vol. 29 (2007).

[4] Nie Chunyan: Chaotic system with weak signal detection, edtied by Tsinghua University Press, Beijing (2007).

[5] Donald L.Birx. Chaotic oscillator and CMFFNS for signal detection in noise environments. IEEE International Joint Conference on Neural Networks, USA, (1992).

[6] Qin Honglei, Sun Xingli, Jin Tian. Weak GPS signal detect algorithm based on duffing chaos system.10th International Conference on Signal Processing. Beijing: IEEE, (2010).

[7] Li Yue, Xu Kai, Yang Baojun, YuanYe, Wu Ning. Chin. Phys. Soc.Vol. 57 (2008).

[8] Li Yue, Shi Yaowu, Ma Haitao,Yang Baojun. Acta Electronica Sinica, Vol.32(2004).

[9] Wang Yongsheng, Jiang Wenzhi, Zhao Jianjun, Fan Hongda. Chin. Phys. Soc. 57(2008).

[10] Guangyu Wang, Dajun Chen, Jiangya Lin. IEEE Transactions on Industrial Electronics. Vol.46(1999).

[11] Li Yue, Yang Baojun: Chaotic Time Around the Systerm (L-Y) and Detection, edtied by Science press, Beijing(2007).

[12] Liu Bingzheng, Peng Jianhua: Nonlinear Dynamics, edtied by High Education Press, Beijing (2004). 